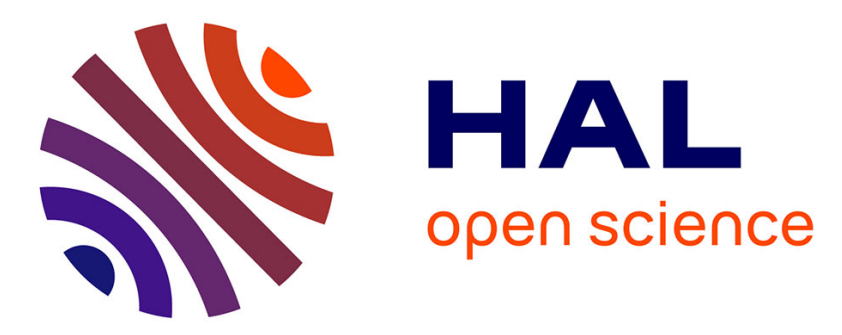

\title{
Micromechanical modelling of the elastic-viscoplastic response of metallic alloys under rapid compression in the semi-solid state
}

Véronique Favier, H. V. Atkinson

\section{- To cite this version:}

Véronique Favier, H. V. Atkinson. Micromechanical modelling of the elastic-viscoplastic response of metallic alloys under rapid compression in the semi-solid state. Acta Materialia, 2011, 59 (3), pp.1271-1280. 10.1016/j.actamat.2010.10.059 . hal-02455183

\section{HAL Id: hal-02455183 \\ https://hal.science/hal-02455183}

Submitted on 25 Jan 2020

HAL is a multi-disciplinary open access archive for the deposit and dissemination of scientific research documents, whether they are published or not. The documents may come from teaching and research institutions in France or abroad, or from public or private research centers.
L'archive ouverte pluridisciplinaire HAL, est destinée au dépôt et à la diffusion de documents scientifiques de niveau recherche, publiés ou non, émanant des établissements d'enseignement et de recherche français ou étrangers, des laboratoires publics ou privés. 


\title{
Micromechanical modelling of the elastic-viscoplastic response of metallic alloys under rapid compression in the semi-solid state
}

\author{
V. Favier ${ }^{\mathrm{a}}$, H.V. Atkinson ${ }^{\mathrm{b}, *}$ \\ ${ }^{a}$ Arts et Métiers ParisTech, PIMM UMR CNRS 8006, ENSAM, 151 Bd de l'Hôpital, 75013 Paris, France \\ ${ }^{\mathrm{b}}$ Department of Engineering, University of Leicester, University Road, Leicester LE1 7RH, UK
}

\begin{abstract}
Semi-solid processing is used commercially to produce a variety of components and it is therefore important to be able to model the die fill. Micromechanical modelling is one approach to this. Here we compare the micromechanical predictions for the load vs. displacement, in tests where a cylindrical billet is rapidly compressed, with previous experimental findings for an A356 aluminium alloy. Purely viscoplastic modelling is shown to be inadequate. We propose a new model that clearly associates the elastic-type response with the saturated solid skeleton. This gives much more accurate prediction of the initial peak and of the form of the curve as the skeleton breaks down under load. In agreement with experiment, the model predicts the time for the solid skeleton breakdown and that the peak load increases with increasing ram speed and with decreasing fraction liquid.
\end{abstract}

Keywords: Semi-solid processing; Aluminium alloys; Elastic behaviour; Micromechanical modelling; Mean field analysis

\section{Introduction}

Semi-solid processing is a method of forming complexshaped components in the semi-solid state. The thixotropic nature of non-dendritic semi-solid alloy and the capacity to exploit this industrially was first realized by Flemings and co-workers at MIT in the early 1970s [1]. Thixotropy means that when the material is sheared it thins and flows, but when it is allowed to stand it thickens again. The processes based on this property are now used industrially to produce a variety of components, particularly from aluminium alloys. The processing is called thixoforming when the material gives a non-dendritic microstructure on reheating into the semi-solid state. In that case, the solid fraction is usually higher than 0.5 . The processing is labelled as rheocasting when a liquid alloy is cooled into

\footnotetext{
* Corresponding author. Tel.: +44 114222 2000; fax: +44 1162522525. son).
}

the semi-semi-solid state and gives a non-dendritic microstructure. In that case, the solid fraction is usually lower than 0.5 . There are a variety of other variants, including thixocasting, thixoforging and rheoforging.

The origin of thixotropy in semi-solid metallic alloys can be understood as follows. The microstructure consists of solid spheroids in a liquid matrix. If the material is allowed to stand, the spheroids agglomerate and the viscosity rises. When the material is sheared the agglomerates are broken up to an extent that depends on the shear rate (Fig. 1 [2]). The viscosity is both time and shear rate dependent, with a dynamic equilibrium between agglomeration and disagglomeration. Each shear rate has a characteristic agglomerate size distribution. When the shear rate is changed, over a period of time, the agglomerate size distribution tends towards that characteristic of the new shear rate, with the viscosity changing commensurately $[3,4]$. In thixoforming, the change in shear rate occurs very rapidly; the billet starts at rest but, within a fraction of a second, as it enters the die cavity it is approximately $100 \mathrm{~s}^{-1}$. Modelling must 


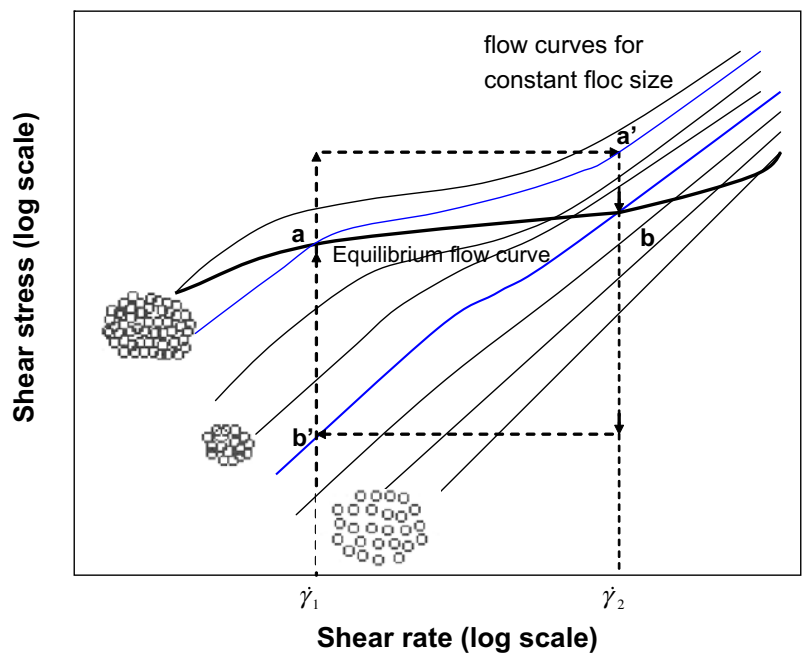

Fig. 1. Flow curves of a flocculated suspension (after [2]).

therefore be appropriate for such rapid changes in shear rate and the experimental validation concerned with transients in behaviour rather than the steady state.

Quaak proposed [5] two characteristic times to represent the response to a jump-up in shear rate. The slurry undergoes an initial rapid breakdown, associated with the breaking of solid-solid bonds followed by a more gradual process of coarsening and spheroidization dependent on diffusion (see Fig. 2). It is the initial rapid breakdown that we are interested in here.

Rapid compression tests (with a fraction solid greater than 0.5) are useful to estimate the semi-solid behaviour in thixoforming [6]. Although the shear regime is more complex than that in a rheometer, rheometers cannot generally be used to characterize material with a fraction of solid greater than about 0.55 . In addition, in rapid compression tests, the rate of compression is of the same order as that in the industrial process and the tests provide the semi-solid response in the transient regime. Modelling the transient behaviour is one of the main challenges in order to perform numerical simulations of optimized thixoforming. Various constitutive equations are proposed in the

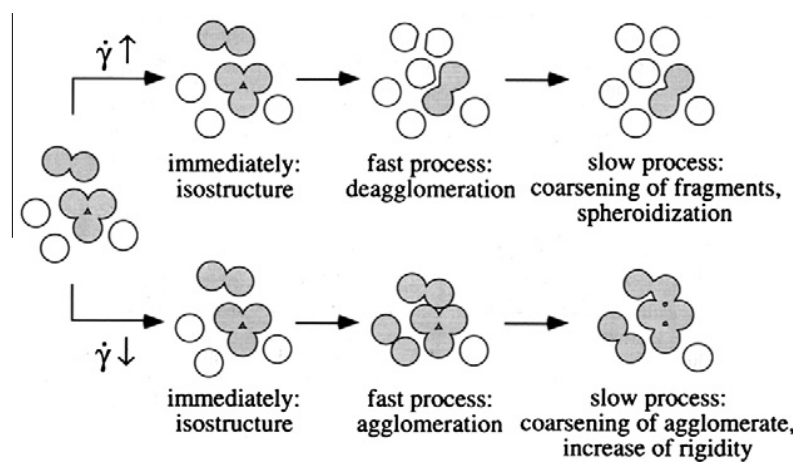

Fig. 2. Schematic model describing the fast and slow processes in a semisolid material's structure after shear rate up and down jumps (taken from Ref. [5]). literature (see e.g. [7]). The thixotropic behaviour is usually accounted for by means of a structure parameter, often designated by $\lambda$. The time derivative of $\lambda$ introduces the rate of build-up (agglomeration) of the solid particles and the rate of breakdown (disagglomeration). Favier et al. [8] proposed a constitutive equation that clarifies the mechanical roles of the liquid and the solid, and of their spatial distribution, within the material in terms of the overall behaviour of semi-solids. To do so, they used micromechanics and homogenization techniques. This modelling successfully described steady-state behaviour (in terms of the order of magnitude and the trends) and was extended to non-steady-state (transient) behaviour $[9,10]$. The present objective is essentially to correlate the mechanical response of semi-solid billet, under compression loading, to the deformation mechanisms and the microstructure of the material. The second section summarizes the basic concepts of the modelling. The third section focuses on the semi-solid billet response under compression loading. The results concerning the influence of ram speed and fraction solid are compared to the experimental results of Liu et al. [11], which were obtained with aluminium alloy A356. In these experiments, samples were heated to the desired temperature in the semi-solid state and rammed at constant velocity against a platen backed by a load cell. When the liquid fraction is relatively low, the load-displacement curves produced from the tests show an initial peak (Fig. 3), which is believed to originate from a skeletal solid structure that breaks down under shear (e.g. [11]). It should be noted that the repeatability on the peak load and on the minimum of the load-displacement curve is $\pm 0.25 \mathrm{kN}$. The load signal during flow decreases with increasing temperature and the initial peak eventually disappears in all the alloys investigated. Quantitative metallography by Liu et al. suggested that the lower loads corresponded to greater spheroidicity of the solid particles within the slurry [11]. The spheroidicity and the temperature are interrelated because the higher the starting temperature the higher the liquid fraction (provided liquid has had time to develop) and the greater the tendency for spheroidization to have occurred. A reasonable guide to the fraction solid present at the beginning of the experiment in Fig. 3 can be obtained from the differential scanning calorimetry results in Fig. 2 of Ref. [12] for A356 aluminium alloy, which suggest that the approximate corresponding solid fractions are: 0.7 at $574{ }^{\circ} \mathrm{C} ; 0.6$ at $576{ }^{\circ} \mathrm{C} ; 0.5$ at $578^{\circ} \mathrm{C}$; and 0.4 at $581{ }^{\circ} \mathrm{C}$.

Experimental evidence of the existence of a solid skeleton has recently been obtained through tomography of semisolid alloy structures (e.g. $[13,14])$. Fig. 4 gives an example. Two figures are given here ( $a$ and $b$ ) because they show different features. Fig. 4a illustrates the potential strength and substantiveness of the interconnected network. Fig. 4b, particularly in the side view, illustrates how, as the solid is compressed, solid-solid bonds could act as hinges during the collapse. Tzimas and Zavaliangos [15] have shown similar results to Liu et al. [11] with stress-strain curves in 


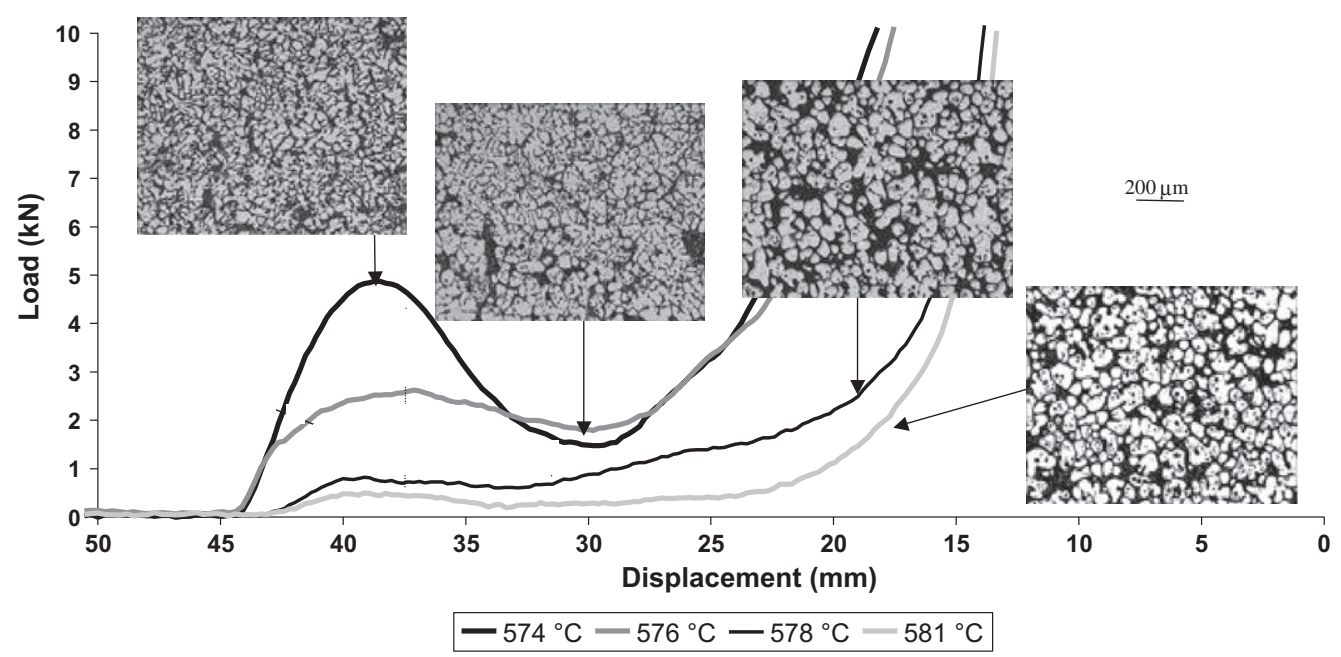

Fig. 3. Load signals and microstructure at different temperatures for Alusuisse A356 Al alloy (ram speed $500 \mathrm{~mm} \mathrm{~s}^{-1}$ and zero soaking time). Microstructures are taken from the edge of the billet after compression has been completed (after [11]).

compression exhibiting a peak at low strain $(5-10 \%)$ and a decrease to a plateau. They argue that the initial peak is due to a combination of elastoplastic deformation at grain contacts and the destruction of cohesive bonds between solid grains.

The Atkinson review of modelling of semi-solid processing [7] showed that, although there is a wide range of modelling approaches in the literature, there are very few published examples of direct comparison between modelling predictions and experimental results (with rare exceptions $[16,17])$. The aim here is to carry out such a comparison and to identify the successes and the shortcomings. The work has potentially wider applicability because there is increasing interest in modelling alloys that are formed above the conventional hot rolling temperature range.

\section{Micromechanical modelling}

\subsection{Viscoplastic analysis}

The micro-macro modelling is based on the following statements (further details are given in Refs. [8-10]):

(1) In semi-solids having a solid fraction $f^{s}$, four mechanical "phases" within the material are identified: the solid globules, the solid bonds between the solid globules (also referred to here as "spheroids"), the free liquid and the liquid entrapped within the solid globules. These phases play different mechanical roles: the deformation mainly takes place in local sites such as the bonds between the solid grains and the liquid that is not entrapped in the agglomerated solid particles. From a statistical point of view, the material is represented via a "coated inclusion" (Fig. 5): the "inclusion" is composed of both solid and liquid with volume fractions $f_{I}^{s}$ and $f_{I}^{l}$ respectively, to represent entrapped liquid within solid particles; the "coating" (the active zone that carries most of the deformation) consists of the solid bonds and the non-entrapped liquid with volume fractions $f_{A}^{s}$ and $f_{A}^{l}$ respectively. $f_{I}$ is the volume fraction of inclusion excluding the active zone and, in effect, gathers together all the phases that do not participate in the deformation. If $f_{A}$ is the volume fraction of the active zone (the zone that mainly accommodates the deformation), then:

$f_{I}+f_{A}=1$

(2) The liquid phase is regarded as a Newtonian fluid (Eq. (2)), whereas the solid phase is considered as viscoplastic (Eq. (3)).

$S_{i j}^{l}=2 \mu^{l} \dot{\varepsilon}_{i j}^{l}$ with $\mu^{l}=K^{l}$

for the Newtonian liquid (suffix $l$ ) and

$S_{i j}^{g, b}=2 \mu^{g, b} \dot{\varepsilon}_{i j}^{g, b}$ with $\left.\mu^{g, b}=K^{g, b} \frac{\sqrt{3} \dot{\varepsilon}_{e q}^{g, b}}{\dot{\gamma}_{0}^{g, b}}\right)^{m^{g, b}-1}$

for the viscoplastic solid globules (suffix $g$ ) and the solid bonds (suffix $b$ ). $\boldsymbol{S}$ and $\dot{\boldsymbol{\varepsilon}}$ are the deviatoric stress and strain rate tensors, respectively, $\mu$ is the viscosity, $\dot{\varepsilon}_{e q}$ is the Von Mises equivalent strain rate. $K$ is the consistency, $m$ is the power-law index (strain rate sensitivity parameter) and $\dot{\gamma}_{0}$ is the reference strain rate taken equal to $1 \mathrm{~s}^{-1}$.

(3) A self-consistent approximation is used at two scales to determine the semi-solid viscosity $\mu_{S S}$. First, the viscosities of the inclusion $\mu_{I}$ and of the active zone $\mu_{A}$, associated with the respective volume fractions $f_{I}$ and $f_{A}$, are calculated from the liquid and solid behaviour using a classical self-consistent scheme. Then the viscosity of the semi-solid is deduced from the inclusion and the active zone viscosities using the self-consistent estimation applied to the specific morphology of the coated inclusion. 
(a)

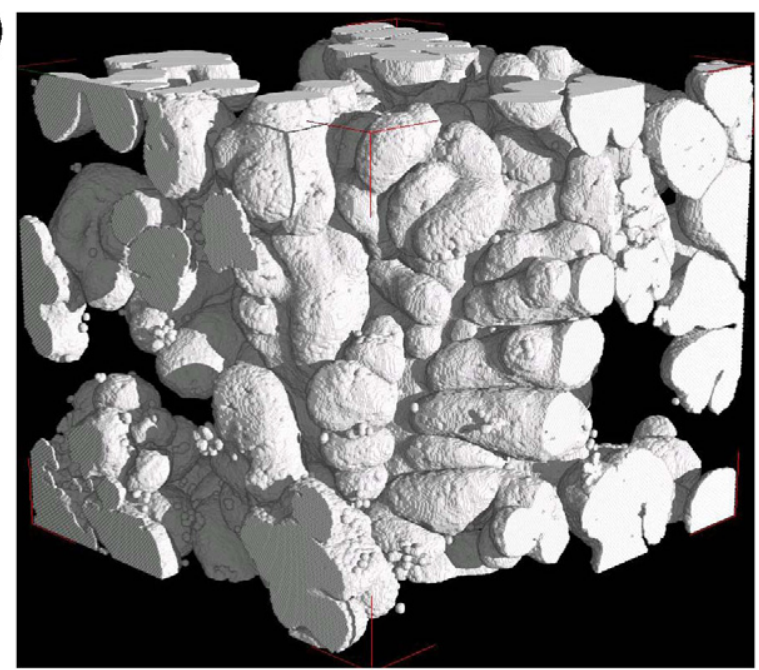

(b)

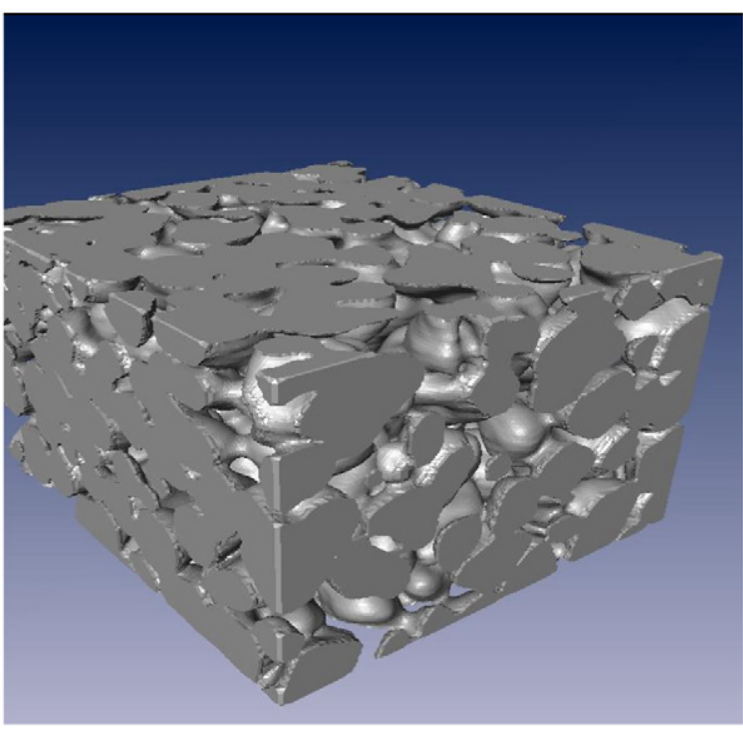

Fig. 4. (a) Three-dimensional interconnected skeleton obtained by phasesensitive synchrotron tomography (in this case with AZ91 alloy, but the phenomenon with A356 aluminium alloy will be similar) [13]. (b) Threedimensional solid network obtained by fast X-ray microtomography with $\mathrm{Al}-15.8 \mathrm{wt} . \% \mathrm{Cu}$ (cooled from the liquid state with approximately $39 \%$ liquid when measured in situ) [14].

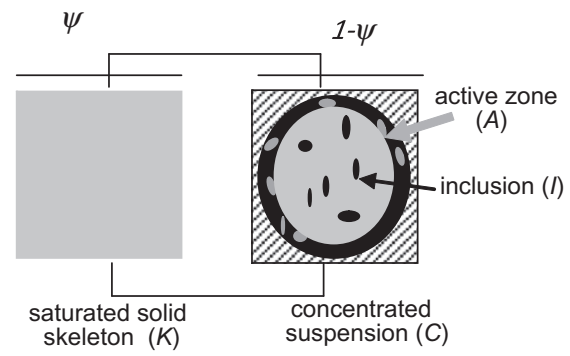

Fig. 5. Schematic representation of the semi-solid for micromechanical modelling with a Voigt model. On the left is the saturated solid skeleton and on the right the "inclusion" of solid and entrapped liquid surrounded by liquid and solid bonds in the "active zone" (after [10]).
(4) The evolution of the microstructure as a function of the strain rate is captured through an internal variable. This internal variable is the solid fraction of the active zone $f_{A}^{s}$ measuring the amount of bonds. The evolution law introduces only disagglomeration owing to shear inside the bonds (Eq. (4)) [10]. This choice is justified by the fact that in rapid compression tests the disagglomeration process dominates over the agglomeration process [7].

$\dot{f}_{A}^{s}=-\left(1-f^{s}\right) \frac{\dot{\gamma}_{\text {bonds }}}{\gamma_{c}} f_{A}^{s}$

The shear rate within the solid bonds $\dot{\gamma}_{\text {bonds }}$ is inherent within the micro-macro modelling and is equal to:

$\dot{\gamma}_{\text {bonds }}=\sqrt{3}\left(\dot{\varepsilon}_{A}^{s}\right)_{e q}$

In addition, we postulate that the solid bonds break as soon as the local shear reaches a critical value $\gamma_{c}$.

It should be emphasized that what has been described so far is essentially a viscoplastic analysis. In Section 3 it will become clear that an elastic or elastic-viscoelastic contribution is also required. This is now described. In the literature, there has been little previous consideration of an elastic contribution to the response to deformation. Modigell and Hufschmidt [18], with oscillation experiments in the semi-solid state, characterize the storage modulus that describes the elasticity of the material. Wahlen [19] and Petera et al. [17] propose equations to model semi-solid behaviour that include an elastic contribution. Here, we propose a new model that clearly associates the elastic-type response of semi-solids with the presence of the saturated solid skeleton.

\subsection{Analysis incorporating elasticity}

As mentioned previously, the solid fraction involved is higher than 0.5. As a result, a solid continuous skeleton exists within the material at rest. In this new analysis incorporating elasticity, we emphasize the role of the solid skeleton at the beginning of the deformation. The semi-solid is represented by a Voigt model having two branches (Fig. 5). The first branch represents the solid skeleton saturated with entrapped incompressible liquid $(K)$. The second branch contains the free liquid and the isolated solid agglomerates. It is labelled as concentrated suspension (C). The fraction of the saturated skeleton is $\psi$. The fraction of the concentrated suspension is $1-\psi$. The choice of the Voigt model is justified by the fact that the deformation is macroscopically homogeneous, at least at the beginning of the deformation, and both branches of the model represent continuous phases. Thus, the average strain rate tensors of $(K)$ and $(C)$ are given by

$\dot{\boldsymbol{\varepsilon}}_{K}=\dot{\boldsymbol{\varepsilon}}_{C}=\dot{\boldsymbol{E}}$

where $\dot{E}$ is the overall strain rate. The overall stress tensor is given by 
$\boldsymbol{\Sigma}=\psi \boldsymbol{\sigma}_{K}+(1-\psi) \boldsymbol{\sigma}_{C}$

where $\sigma_{K}$ and $\sigma_{C}$ are the average stress tensors of the saturated skeleton and the concentrated suspension, respectively. When a semi-solid deforms, the solid skeleton breaks down up to the complete failure of the three-dimensional (3-D) solid network. As a result, at the end of the deformation, the semi-solid appears as a suspension composed of isolated solid agglomerates (provided the solid fraction is not too high, i.e. less than about 0.9 ).

From a modelling point of view, these changes are represented by the change of $\psi$ with the overall strain and strain rates as follows:

$\dot{\psi}=-D_{K} \psi\left(1-f^{S}\right) \dot{E}_{e q} E_{e q}$

where $D_{K}$ is constant and characterizes the disagglomeration rate of the solid skeleton. The initial value of $\psi$ is assumed to be given by

$f^{s}<f^{c} ; \quad \psi_{\text {init }}=0$

$f^{c} \leq f^{s}<1 ; \quad \psi_{\text {init }}=1-\frac{f^{c}}{f^{s}}$

$f^{s}=1 ; \quad \psi_{\text {init }}=1$

where $f^{c}$ is the percolation threshold prescribed at 0.4 $[13,14]$.

The saturated skeleton is assumed to have an elasticviscoplastic behaviour:

$\dot{\boldsymbol{\sigma}}_{K}=\boldsymbol{c}_{K}:\left(\dot{\boldsymbol{\varepsilon}}_{K}-\dot{\boldsymbol{\varepsilon}}_{K}^{v p}\right)$

where $c_{K}$ is the elastic moduli tensor (assumed isotropic) and $\dot{\boldsymbol{\varepsilon}}_{K}^{v p}$ is the viscoplastic strain rate tensor of the saturated skeleton.

The viscoplastic behaviour is assumed incompressible and is given by a power-law type equation:

$\left.\boldsymbol{S}_{K}=2 \mu_{K} \dot{\boldsymbol{\varepsilon}}_{K}^{v p}=2 K_{K} \frac{\sqrt{3} \dot{\varepsilon}_{e q, K}^{v p}}{\dot{\gamma}_{0}}\right)^{m_{K}-1} \dot{\boldsymbol{\varepsilon}}_{K}^{v p}$

where $\boldsymbol{S}_{K}$ is the deviatoric part of the stress tensor, $\dot{\varepsilon}_{e q, K}^{v p}$ is the Von Mises equivalent viscoplastic strain rate, $K_{K}$ is the consistency and $m_{K}$ is the strain rate sensitivity parameter associated with the saturated skeleton. $\dot{\gamma}_{0}$ is a reference slip rate equal to $1 \mathrm{~s}^{-1}$.

The concentrated suspension is assumed to behave as a non-Newtonian fluid:

$\boldsymbol{S}_{C}=2 \mu_{C} \dot{\boldsymbol{\varepsilon}}_{C}$

where $\mu_{C}$ is the viscosity of the concentrated suspension. This viscosity is given by the previous micro-macro model [7]. Note that, for the concentrated suspension, the entrapped liquid is trapped inside the solid spheroids and agglomerates (not inside the continuous 3-D solid skeleton): both constitute the inclusion. The viscoplastic deformation is assumed to be accommodated by the solid bonds within the solid isolated agglomerates and the free liquid (the active zone).
Assuming that the volumes of liquid and solid do not change, the following equations have to be checked

$f^{s}=\frac{V_{K}^{s}}{V}+\frac{V_{C}^{s}}{V}$

$f^{l}=1-f^{s}=\frac{V_{K}^{l}}{V}+\frac{V_{C}^{l}}{V}$

$V$ is the volume of the representative volume element. $V_{K}^{s}$ and $V_{C}^{s}$ are the volumes of solid belonging to the skeleton and to the concentrated suspension, respectively. $V_{K}^{l}$ and $V_{C}^{l}$ are the volumes of liquid belonging to the skeleton and to the concentrated suspension, respectively. We can easily demonstrate that all these quantities can be expressed as a function of $\frac{V_{K}^{s}}{V}, \psi$ and $f^{s}$.

We assume that

$\frac{V_{K S}}{V}=\psi f^{S}$

Eq. (15) captures the fact that, when $\psi$ or $f^{s}$ increases, the amount of solid belonging to the skeleton increases. As mentioned in the introduction, the breakdown of the solid 3-D skeleton is followed by a disagglomeration process of the solid agglomerates, leading to a suspension having more and more isolated solid spheroids. To capture this microstructural change, we assume that the rate of change of amount of solid bonds between the isolated agglomerates, $\dot{f}_{A}^{s}$, follows Eq. (4). It should be noted that any elastic contribution is only operating over a few milliseconds at the beginning of the test. In addition, the modulus is relatively low (in the region of $\sim 50 \mathrm{MPa}$ ), as would be expected with an aluminium alloy in this temperature range.

\section{Results and discussion}

\subsection{Simulations of compression tests}

We simulated compression tests using the micro-macro model assuming uniform and uniaxial stress fields. In this section, model parameters were chosen to be qualitatively consistent with the experimental results of Liu et al. [11]. We emphasize that at this stage in the paper we are examining general trends rather than obtaining detailed validation of the modelling. In the next section, a more quantitative analysis will be presented. We consider solid fractions higher than 0.5 to be in agreement with usual compression tests. Since the solid fraction is quite high and the material may be at rest prior to the application of the load, we assume that there is a continuous solid skeleton initially within the material. More precisely, we followed the test parameters of Ref. [11]. The ram speed used was $500 \mathrm{~mm} \mathrm{~s}^{-1}$, unless otherwise stated. The height and diameter of the initial slug in the experiments are 42 and $36 \mathrm{~mm}$, respectively. During the experiment, as the slug heats up, it expands to a height of $44 \mathrm{~mm}$, so this value has been used for the simulation. There is no lateral constraint either in the experiments or in the simulation, i.e. the material is free to flow sideways. Fig. 6 displays the 


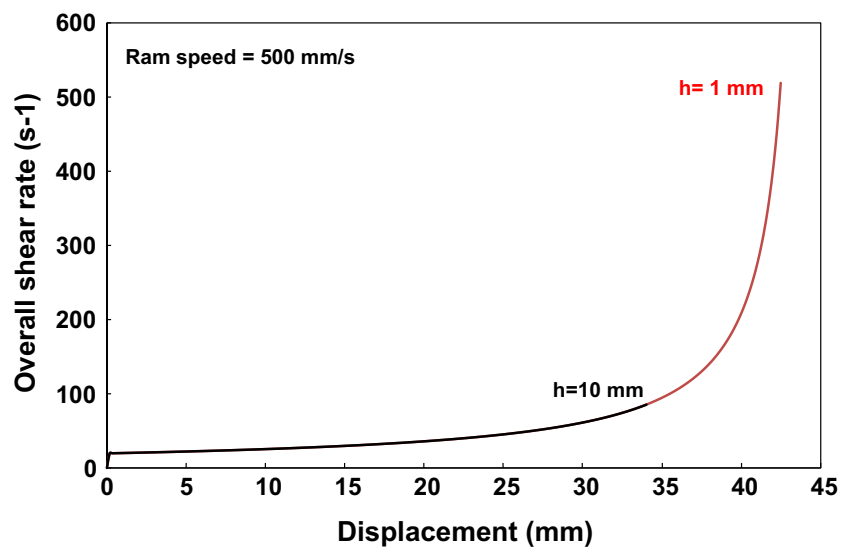

Fig. 6. Modelled evolution of the overall equivalent shear rate during a uniaxial compression test at a ram speed of $500 \mathrm{~mm} \mathrm{~s}^{-1} . h$ is the remaining height during compression.

evolution of the overall equivalent shear rate vs. the specimen displacement throughout the test. The overall equivalent shear rate immediately after displacement starts is $20 \mathrm{~s}^{-1}$. It rises to $90 \mathrm{~s}^{-1}$ by the time the height has been compressed to $10 \mathrm{~mm}$ (i.e. $34 \mathrm{~mm}$ on the axis) and to just over $500 \mathrm{~s}^{-1}$ when the billet is only $1 \mathrm{~mm}$ thick $(43 \mathrm{~mm}$ displacement). Examining the experimental results in Fig. 3 (and note that these are for load vs. displacement rather than for strain rate and that the first contact with the die is on the left-hand side of the plot, where the displacement axis in this case reads $44 \mathrm{~mm}$ ), the billet does not reach this degree of compression before the load rises very steeply in the final stages of the test. One of the challenges here is to account for this steep rise at this stage of the compression. It may be associated with friction; we consider here in the modelling only pure uniaxial compression, whereas we do not have pure uniaxial compression in experiments because of friction between the billet and the platen. The occurrence of friction increases the strain rate locally (classically there is a "cross pattern" in the billet under compression where the strain rate is locally higher along the diagonals). The role of friction merits further investigation. In addition, solidification may be occurring in the final stages of the test, leading to the steep rise, but the model here is isothermal.

\subsection{Comparison of predicted and experimental load- displacement curves}

The solid fraction associated with the experiments described in Ref. [11] varies depending on the starting temperature and the particular aluminium alloy starting material. As a first approach, we consider that the solid fraction is 0.7 . Model parameters were chosen to be qualitatively consistent with experimental results of Ref. [11] for this fraction solid. Parameters associated with the material representation are listed in Table 1, unless otherwise stated. Rheological parameters are listed in Table 2 and remain constant for all the subsequent simulations.
Table 1

Parameters associated with the representation of the material.

\begin{tabular}{llll}
\hline$f_{\text {Ainitial }}^{s}$ & $\gamma_{c}$ & $f_{A}$ & $f_{c}$ \\
\hline 0.417 & 1.0 & 0.04 & 0.4 \\
\hline
\end{tabular}

Table 2

Rheological parameters.

\begin{tabular}{llll}
\hline Solid & & & Liquid \\
\hline$K^{g}(\mathrm{~Pa} \mathrm{~s})$ & $K^{b}(\mathrm{~Pa} \mathrm{~s})$ & $m^{g}=m^{b}$ & $K^{l}(\mathrm{~Pa} \mathrm{~s})$ \\
\hline $1.7 \times 10^{6}$ & $1.7 \times 10^{6}$ & 0.2 & $1.8 \times 10^{-3}$ \\
\hline
\end{tabular}

Note that the spheroidicity or otherwise of the solid particles is incorporated into the consistency of the solid phase.

\subsubsection{Viscoplastic analysis: effect of ram speed}

First of all, the effect of ram speed will be examined. Fig. 7 shows the prediction and Fig. 8 the experimental results from Ref. [11]. As in the experiment, the prediction shows that the height of the initial peak increases with ram speed. Here we look for the occurrence of an initial peak and the general form rather than the precise quantitative results. Typically, the load-displacement curve increases up to a maximum and thereafter strongly decreases and increases again, as experimentally observed. The flow load after the peak (i.e. the minimum of the curve) represents the value for the flow of a suspension of agglomerates where few bonds are now being broken. Indeed, the modelling can identify at what point the material becomes a suspension of agglomerates (when the solid fraction in the active zone has decreased below the percolation limit) and when there is finally no more break-up of agglomerates. These points are indicated in Fig. 7 (and are dependent on ram speed). The predicted curves do not show the "bulge" that some of the experimental curves show just before the load-displacement curves converge. In addition, the predicted curves do not converge. The "bulge" could be associated with the inhomogeneous strain rate field and

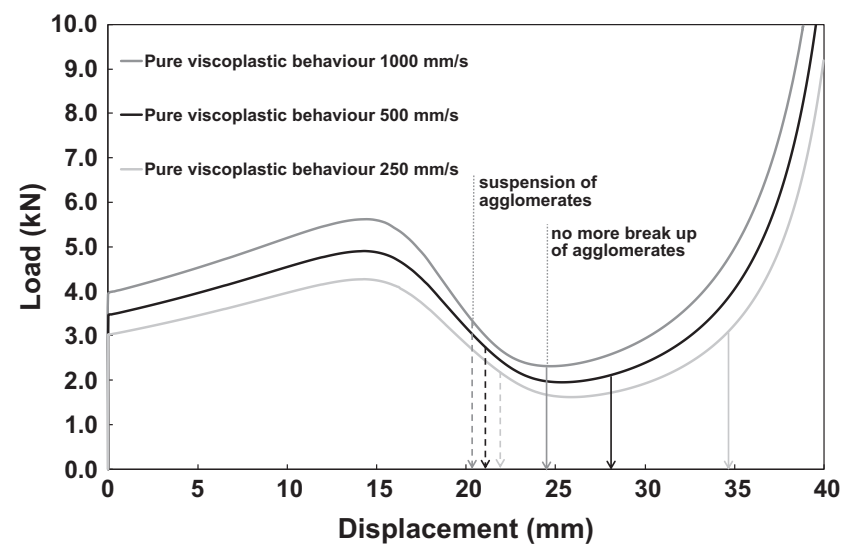

Fig. 7. Predicted load-displacement curves for ram speeds of 1000, 500 and $250 \mathrm{~mm} \mathrm{~s}^{-1}$ (after [9]). A356 alloy at 0.7 fraction solid. 


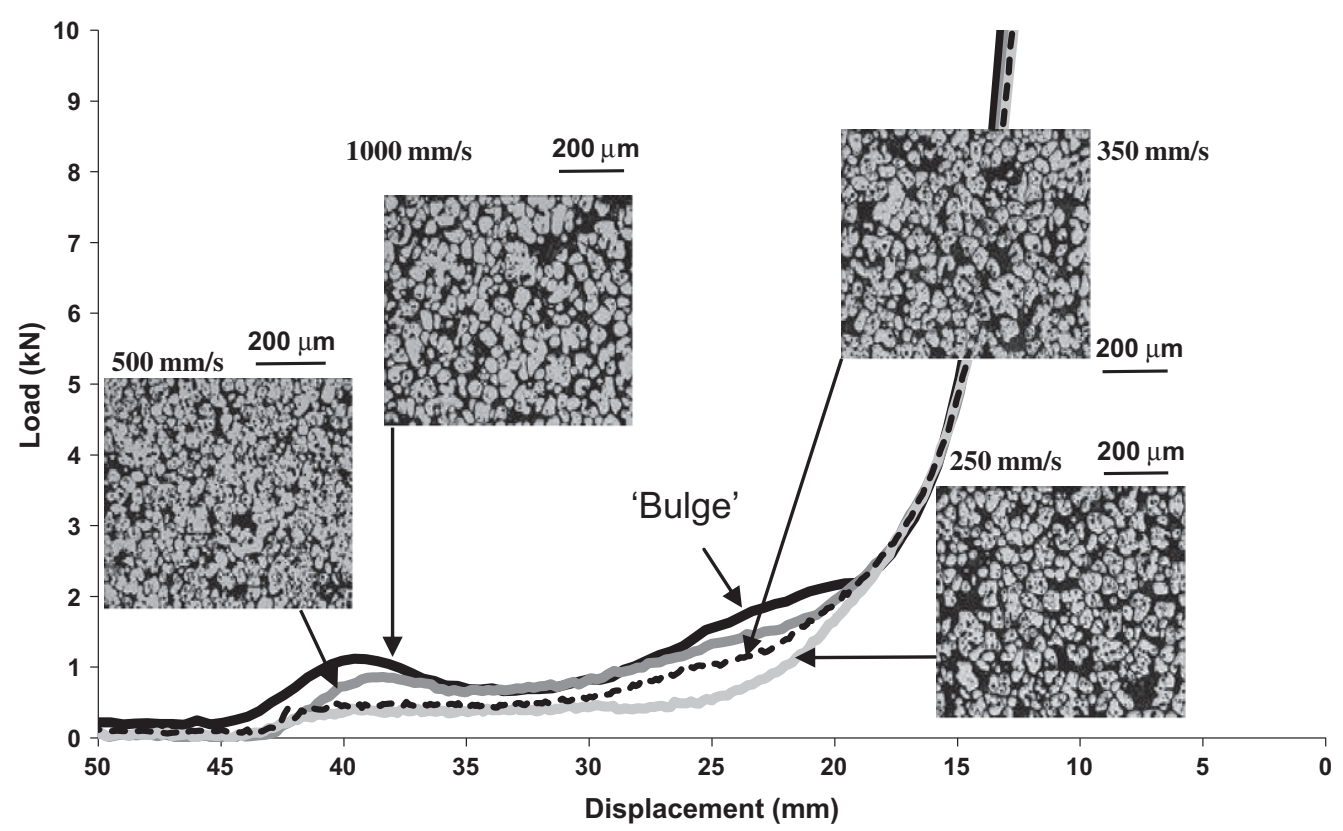

Fig. 8. Experimental load-displacement curves for various ram speeds (after [11]). A356 at $576{ }^{\circ} \mathrm{C}$ and 0 soak. Microstructures are taken from the edge of the billet after compression has been completed.

blocks of material, where disagglomeration has not fully occurred, being compressed in the later stages of the test.

The most significant difference between the predictions and the experiments is in the initial parts of the curves. On the experimental curve the displacement is plotted having zeroed the ram against the platen before the experiment starts. The zero is therefore on the right hand side. The billet first contacts the platen at the $\sim 44 \mathrm{~mm}$ position on the horizontal axis. The initial peak occurs within the first few millimetres of displacement after contact between the billet and the platen. The initial rise of the experimental curve to the peak is steep. In the predicted curves, the peak occurs at about $15 \mathrm{~mm}$ displacement after a much slower rise. There is also an initial offset on the vertical axis. If these offsets were subtracted from the predicted curves, the peak heights would be very close indeed to the experimentally found values. The offset, slow rise and incorrect horizontal peak position in the prediction are essentially associated with the viscoplastic nature of the analysis in this part of the curve. This therefore raises the question of whether the initial breakdown might involve far more elastic or viscoelastic behaviour than might be expected from conventional considerations.

The existence of a yield stress in thixotropic semi-solid alloys is a matter of controversy (e.g. [21,22]). Barnes et al. $[23,24,2]$ have argued that, although a yield stress may be found by workers, this could be due to limitations in their experimental apparatus in not being able to measure shear stresses at very low shear rates. However, using a shear-stress-controlled rheometer, Koke and Modigell [25] have measured a yield stress in $\mathrm{Sn} 15 \% \mathrm{~Pb}$. They distinguish between a static yield stress, where the fluid is at rest prior to the application of the shear stress, and, if the fluid is being continuously sheared, a dynamic yield stress. A number of modellers of semi-solid processing have used a yield stress in their calculations (see the tables in Ref. [7] for a summary). It is incontrovertibly true that a peak is found in the experiments here when there are relatively low liquid fractions, i.e. when the temperature is relatively low. If this does correspond to a yield stress, then it would be equivalent to a static yield stress as the fluid is starting from a rest position before being brought into contact with the platen.

To take account the possibility of the existence of an elastic contribution, an elastic-viscoplastic model has been developed as described earlier.

\subsection{Comparison of experimental and predicted load- displacement curves using elastic-viscoplastic modelling}

\subsubsection{Changes in the predicted response}

The parameters used for the elastic-viscoplastic modelling are given in Tables 3 and 4 .

Fig. 9 compares the experimental load-displacement curve with the calculated curves assuming elastic-viscoplastic behaviour for the semi-solid and comparing with the pure viscoplastic analysis. Very good agreement with the experiment is now obtained for the early stages of the test, i.e. the initial peak. We no longer have the offset,

\section{Table 3}

Set of parameters associated with the representation of the material used for elastic-viscoplastic simulations of compression tests.

\begin{tabular}{lllll}
\hline$f_{\text {Ainitial }}^{s}$ & $D_{K}$ & $\gamma_{c}$ & $f_{A}$ & $f^{c}$ \\
\hline 0.401 & $200 \mathrm{~s}^{-1}$ & 1 & 0.04 & 0.4 \\
\hline
\end{tabular}


Table 4

Set of constitutive parameters used for elastic-viscoplastic simulations of compression tests.

\begin{tabular}{|c|c|c|c|c|c|c|}
\hline \multicolumn{4}{|l|}{ Skeleton } & \multicolumn{2}{|l|}{ Solid } & \multirow{2}{*}{$\begin{array}{l}\text { Liquid } \\
K^{l}(\mathrm{~Pa} \mathrm{~s})\end{array}$} \\
\hline$E_{K}(\mathrm{~Pa})$ & $K^{K}(\mathrm{~Pa})$ & $m^{K}$ & $K^{g}(\mathrm{~Pa} \mathrm{~s})$ & $K^{b}(\mathrm{~Pa} \mathrm{~s})$ & $m^{g}=m^{b}$ & \\
\hline $1 \times 10^{8}$ & $2 \times 10^{7}$ & 0.2 & $1.7 \times 10^{6}$ & $1.7 \times 10^{6}$ & 0.2 & $1.8 \times 10^{-3}$ \\
\hline
\end{tabular}

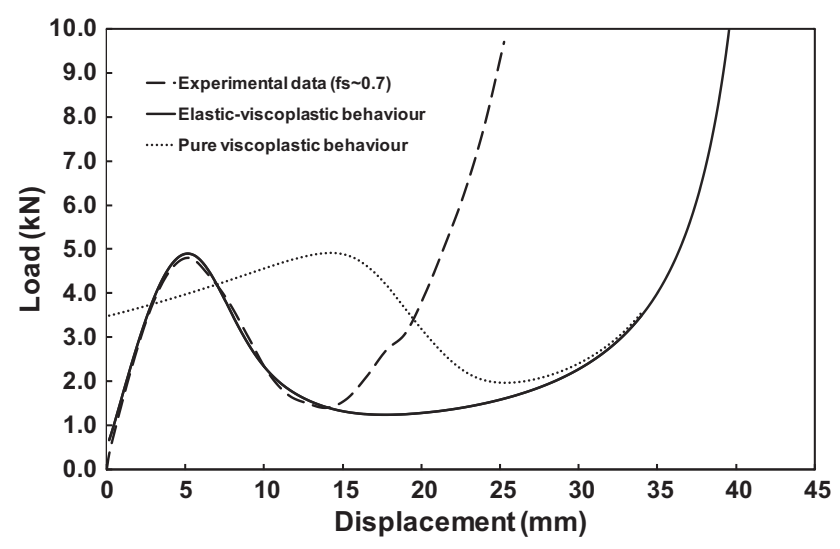

Fig. 9. Experimental and predicted load-displacement curves comparing the elastic-viscoplastic model, the pure viscoplastic model (both with fraction solid of 0.7) and experimental results [11] for Alusuisse A356 alloy at $574^{\circ} \mathrm{C}$ and a ram speed of $500 \mathrm{~mm} \mathrm{~s}^{-1}$.

the low initial slope or the incorrect horizontal peak position. At the start of the compression, the load is mainly carried by the saturated skeleton. In the later stages of the test, the elastic-viscoplastic analysis and the experimental results diverge, with the experimental curve rising steeply earlier than in the model. The potential reasons for this (friction and/or solidification) have been discussed earlier and the model would require further development to deal with these. In the later stages of the deformation, bonds of the solid skeleton break down, some liquid is released, and more and more isolated solid agglomerates are created. As a result, the contribution of the concentrated suspension increases and the overall behaviour becomes mainly viscoplastic. Fig. 10 illustrates this, showing the stress

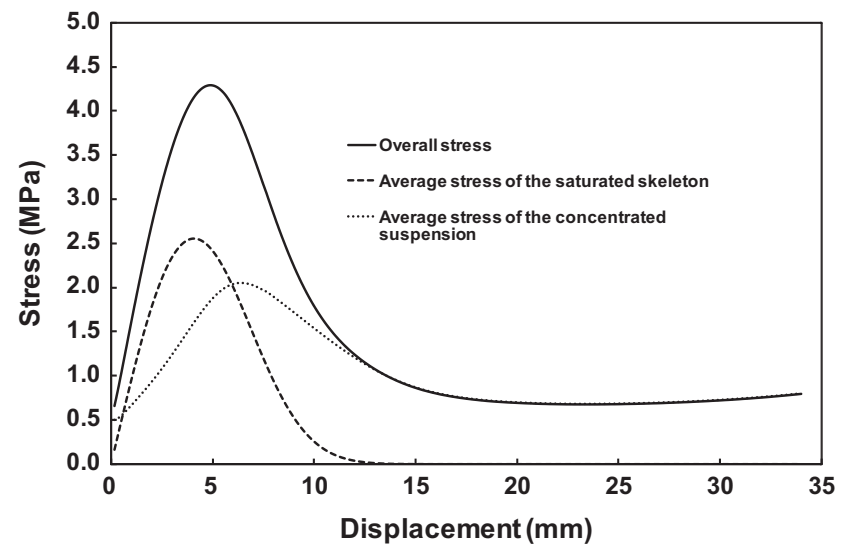

Fig. 10. Predicted average local stresses vs. overall displacement assuming elasto-viscoplastic semi-solid behaviour (ram speed $500 \mathrm{~mm} \mathrm{~s}^{-1}$ ). contributions of the saturated skeleton and the concentrated suspension separately, along with the overall stress.

\subsubsection{Elastoviscoplastic modelling: effect of ram speed}

Fig. 11 shows the influence of ram speed on the results for the elastic-viscoplastic modelling. The modelling correctly picks up that the initial experimental slope $[11,15]$ does not depend on the ram speed, but the initial peak height does. Given the uncertainty in the fraction of solid that should be used for the modelling (the value of 0.6 has been used for Fig. 11, but note that this is the only change in the input parameters; there has been no "refitting"), the peak loads predicted and the flow loads after the peak (i.e. the minimum of the curve) are of the correct order of magnitude. (Note that the parameter fitting has been carried out with the $500 \mathrm{~mm} \mathrm{~s}^{-1}$ curve.) The peak increases as the ram speed increases because of the strain rate sensitivity ( $m$ in the analysis). In addition, the modelling correctly captures the increase in the minimum of the curve with the increase in the ram speed.

The width of the initial peak is about $10 \mathrm{~mm}$ displacement for the predicted $1000 \mathrm{~mm} \mathrm{~s}^{-1}$ curve (Fig. 11). This gives a time for the breakdown of the solid skeleton of approximately $10 \mathrm{~ms}$, which is in very good agreement with that found experimentally. The model is therefore capturing the rate of breakdown of the skeleton quite accurately. If this breakdown time is compared with that found in concentric cylinder rheometry experiments (e.g. [20], albeit for $\mathrm{Sn} 15 \% \mathrm{~Pb}$ rather than aluminium alloy) it is found to be at least an order of magnitude different. The experiments in

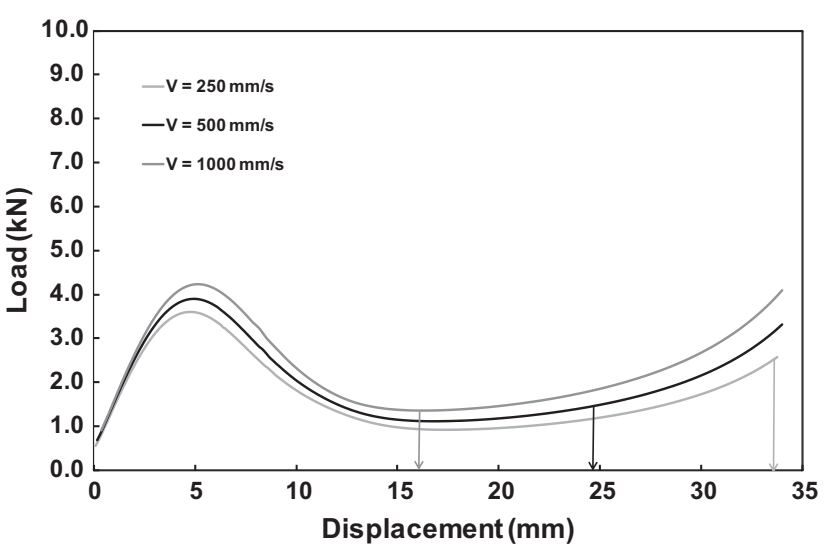

Fig. 11. Predicted load-displacement curves assuming elastic-viscoplastic semi-solid behaviour for ram speeds of 1000,500 and $250 \mathrm{~mm} \mathrm{~s}^{-1}$. The solid fraction in this case is 0.6 to correlate with the experimental results in Fig. 8. The arrows represent the point where, for each ram speed, no further disagglomeration occurs. 
Ref. [20] are for rapid changes in shear rate, including those where the jump is from one finite shear rate to another, i.e. where no solid skeleton is likely to exist. It is therefore thought that the short breakdown time $(\sim 10 \mathrm{~ms})$ found in the rapid compression experiments represents the breakdown of the solid skeleton, whereas the longer one (100$200 \mathrm{~ms}$ ) found in the rheometry experiments is essentially associated with the break-up of agglomerates. If this latter breakdown time is compared with the experimental results in displacement terms (i.e. accounting for the ram velocity), it will be seen that agglomerate breakdown is still occurring in the "minimum" part of the experimental curve.

\subsubsection{Elastoviscoplastic modelling: effect of fraction solid}

We now examine the effect of solid fraction (i.e. starting temperature). The experimental results have already been presented in Fig. 3. The predictions for fractions solid of 0.6, 0.7 and 0.8 are shown in Fig. 12. The model predicts that the height of the peak falls with decreasing solid fraction (or increasing temperature) and the minimum in the load curve also falls. In addition, the peak shifts slightly to the left as the fraction solid decreases. In the experimental curves, the two highest temperature samples (578 and $581{ }^{\circ} \mathrm{C}$ ) may well have slumped slightly, explaining why the "first contact" with the platen (i.e. where the curve starts to rise from the horizontal) is at about $42 \mathrm{~mm}$ on the horizontal axis rather than the $44 \mathrm{~mm}$ for the two lower temperature samples. If these former two curves are shifted to the left so that the contact point is the same, there would be some evidence for a shift to the left in the peak as the solid fraction decreases. The slope on the left-hand side of the peak would be lower for the two higher temperature samples than for the two lower temperature ones. For the predictions, the slope on the left-hand side of the peak also decreases with increasing temperature. This would be consistent with the volume fraction of the skeleton decreasing as the temperature increases. The fraction solid for the experimental curves is not given in Ref. [11] because it is very difficult to obtain with any accuracy. The equilibrium fraction of liquid takes time to develop even once the

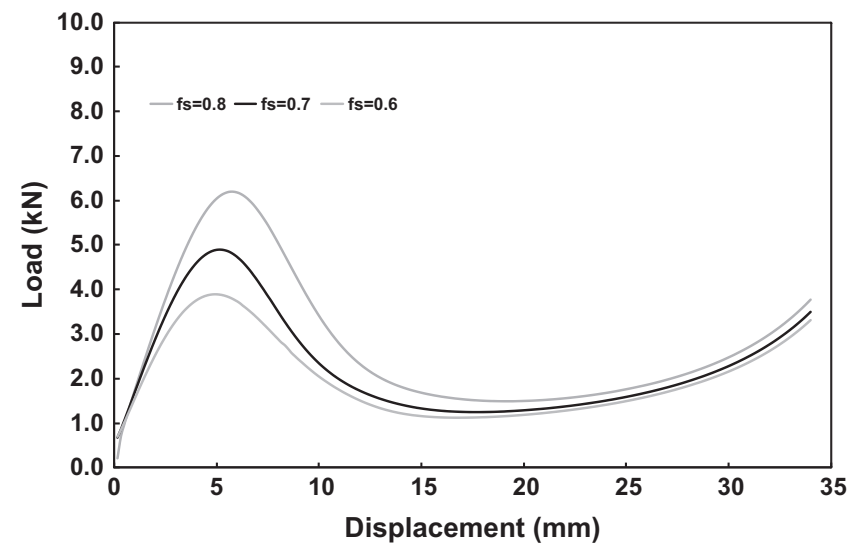

Fig. 12. Predicted load-displacement curves for isothermal compression tests for different solid fractions (ram speed of $500 \mathrm{~mm} \mathrm{~s}^{-1}$ ). required temperature has been achieved in the induction heater, so the temperature itself may not be a good guide. The use of metallographic evidence (e.g. the microstructures shown in Fig. 3) is also flawed as there is a tendency during the cooling of the sample (even if that is quite rapid) for further solid to deposit during the quench, hence making it appear as though there was more solid present at temperature than there actually was (see [14]).

\section{Conclusions}

Semi-solid processing is used commercially to produce a variety of components and it is therefore important to be able to model the die fill. Micromechanical modelling is one approach to this. Here we have compared the micromechanical predictions for the load vs. displacement, in tests where a cylindrical billet is rapidly compressed, with previous experimental findings for an A356 aluminium alloy for a variety of ram speeds and solid fractions. The experiments show an initial peak in the curve, followed by a minimum and then a steep rise towards the end of compression. Purely viscoplastic modelling is shown to be inadequate, with an initial offset on the load axis that does not exist in experiment, incorrect horizontal positioning of the initial peak and a slope of the initial part of the curve that is too low. An elastic component is therefore introduced into the analysis which then gives much more accurate prediction of the initial peak and of the form of the curve, but with the final steep rise occurring later than in the experiment. This may be due to either friction between the billet and the platen or progressive solidification during the course of the test; the experiments are not isothermal. This requires further investigation.

The existence of an elastic component is significant because there has been very little consideration in the literature of the possibility of an elastic contribution in the response of metallic alloys to deformation in the semi-solid state. The elastic contribution is thought to originate from the saturated skeleton of solid. This skeleton breaks down under load, resulting in a peak load. For the same initial semi-solid structure, the model predicts that increasing the ram speed increases the peak load because of a strain rate effect. In addition, the peak load decreases as the volume fraction of liquid in the starting microstructure increases. Both effects correspond with experimental results. The time for the solid skeleton breakdown is also found to be in good agreement with such results.

Overall, the new model has shown that it can predict important features of the experimental results, including trends in the effect of the ram speed and fraction solid, with only one initial "fitting" step, i.e. there is no refitting when, for example, the fraction solid is changed.

\section{Acknowledgements}

We would like to acknowledge the generous assistance of the Royal Society of the United Kingdom, Arts et 
Métiers ParisTech (ENSAM) and the EU Project COST 541 for the funding that enabled this work to be carried out. It builds on earlier work funded by the Engineering and Physical Sciences Research Council of the UK, and we would like to acknowledge here the kind permission from H.V.A.'s co-authors on Ref. [11] to use results from that paper in the modelling presented here.

\section{References}

[1] Spencer DB, Mehrabian R, Flemings MC. Metall Trans 1972;3:1925.

[2] Barnes HA. J Non-Newton Fluid Mech 1997;70:1.

[3] Kumar P, Martin CI, Brown S. Acta Metall Mater 1994;42:3595.

[4] Martin CI, Kumar P, Brown S. Acta Metall Mater 1994;42:3603.

[5] Quaak CJ. PhD thesis, Technische Universiteit Delft; 1996.

[6] Kapranos P, Liu TY, Atkinson HV, Kirkwood DH. J Mater Process Technol 2001;111:31.

[7] Atkinson HV. Progr Mater Sci 2005;50:341.

[8] Favier V, Rouff C, Bigot R, Berveiller M, Robelet M. Int J Form Process 2004;7:177.

[9] Favier V, Manceau M. Int J Mater Form 2009;2(Suppl. 1):737.

[10] Favier V, Cézard P, Bigot R. Mater Sci Eng A 2009;517:8.

[11] Liu TY, Atkinson HV, Kapranos P, Kirkwood DH, Hogg SC. Metall Mater Trans A 2003;34:1545.
[12] Liu D, Atkinson HV, Jones H. Acta Mater 2005;53:3807.

[13] Zabler S, Haibel A, Lohmüller A, Banhart J, Rueda A, Rack A, et al. ECNDT 2006-We.1.5.2. <http://www.ndt.net/article/ecndt2006/doc/ We.1.5.2.pdf>.

[14] Ludwig O, DiMichiel M, Falus P, Salvo L, Suery M. In: 8th international S2P conference on semi-solid processing of alloys and composites, limassol, cyprus, NADCA, Wheeling, IL, 21-23 September, 2004 [CD-ROM].

[15] Tzimas E, Zavaliangos A. Acta Mater 1999;47:517.

[16] Hufschmidt M, Modigell M, Petera J. J Non-Newton Fluid Mech 2006;134:16.

[17] Petera J, Kaminski K, Kotynia M. Int J Mater Form 2010;3:775.

[18] Modigell M, Hufschmidt M. Solid State Phenom 2006;116-117:587.

[19] Wahlen A. Mater Sci Forum 2002;396-402:185.

[20] Liu TY, Atkinson HV, Ward PJ, Kirkwood DH. Metall Mater Trans A 2003;34:409.

[21] McLelland ARA, Henderson NG, Atkinson HV, Kirkwood DH. Mater Sci Eng A 1997;232:110.

[22] de Figueredo A, editor. Science and technology of semi-solid metal processing. Rosemont (IL): North American Die Casting Association; 2001 [chapter 5].

[23] Barnes HA, Walters K. Rheol Acta 1985;24:323.

[24] Barnes HA, Hutton JF, Walters K. An introduction to rheology. Amsterdam: Elsevier; 1989.

[25] Koke J, Modigell M. J Non-Newton Fluid Mech 2003;112:141. 